Article

\title{
Development of a Segmental Bioelectrical Impedance Spectroscopy Device for Body Composition Measurement
}

\author{
Thomas Cannon and JungHun Choi *(D) \\ Department of Mechanical Engineering, Georgia Southern University, Statesboro, GA 30458, USA; \\ tc05196@georgiasouthern.edu \\ * Correspondence: jchoi@georgiasouthern.edu
}

Received: 4 October 2019; Accepted: 1 November 2019; Published: 6 November 2019

check for updates

\begin{abstract}
Whole-body bioelectrical impedance analysis for measuring body composition has been well-explored but may not be sensitive enough to changes in the trunk compared to changes in the limbs. Measuring individual body segments can address this issue. A segmental bioelectrical impedance spectroscopy device (SBISD) was designed for body composition measurement and a prototype was implemented. Compensation was performed to adjust the measured values to correct for a phase difference at high frequencies and to counteract the hook effect when measuring the human body. The SBISD was used to measure five subjects and was compared against three existing analyzers. For most segmental measurements, the SBISD was within $10 \%$ of the $R_{0}$ and $R_{\infty}$ values determined with a Bodystat Multiscan 5000 and an Impedimed SFB7. The impedance values from the third reference device, a Seca 514, differed significantly due to its eight-electrode measuring technique, meaning impedance measurements could not be compared directly.
\end{abstract}

Keywords: bioelectrical impedance spectroscopy; bioimpedance; segmental; BIS; BIA; body composition

ClinicalTrials.gov Identifier: NCT04006899. Retrospectively registered 1 July 2019 at https://clinicaltrials. gov/ct2/show/NCT04006899

\section{Introduction}

Measuring and tracking body composition can help to assess and track the health of an individual in areas such as nutrition, hydration, and recovery monitoring [1]. Bioelectrical impedance analysis (BIA) has been explored to address these topics. This technique measures the impedance of the body, typically from wrist to ankle, when subjected to a small current injected into the body. Typically, this current is applied with gel tab electrodes or hand grips and foot pads. Several technologies for applying bioelectrical impedance analysis have been developed, differing in the number of frequencies the impedance is measured at, the number of electrodes used, and the sections of the body that are measured. Bioelectrical impedance spectroscopy (BIS) involves taking impedance measurements at many frequencies. BIS, particularly when analyzing individual body segments, is a BIA technique that has not been fully explored, and could allow for a noninvasive, quick, portable, and relatively inexpensive method for determining and tracking body composition. The resistance and reactance at each frequency are plotted and then fitted with a curve, called a Cole-Cole curve, to allow for extrapolation of the body resistance at a theoretical zero frequency, $R_{0}$, and the body resistance at a theoretical infinite frequency, $R_{\infty}[1]$. 


\subsection{Pros and Cons of Bioelectrical Impedance Analysis Methods}

Compared to many reference methods, bioelectrical impedance analysis methods, such as BIS, are noninvasive, safe, portable, inexpensive, and repeatable, making them ideal for tracking changes [2,3]. It just requires the placement of electrodes on the subject. It has advantages over other techniques, such as dilution methods, dual-energy X-ray absorptiometry (DXA), and air displacement plethysmography (ADP). Dilution techniques require blood samples. Methods like DXA require the baby to be exposed to radiation. BIS is relatively quick and convenient compared with ADP, another measurement option [3]. BIS was found to be capable of measuring the body composition of infants without turning off monitoring equipment in the intensive care unit (ICU) with good repeatability [4]. In that study, the data points at $52.9 \mathrm{kHz}$, near the operating frequency of a cardio-respiratory monitor, and $26.4 \mathrm{kHz}$ were slight outliers, but with the many frequencies measured, it did not affect results. Single frequency devices would not be capable for such an application due to their focus on the $50 \mathrm{kHz}$ frequency. A survey of BIA studies found that it provides excellent reproducibility for body fat percentage (\%BF) estimations, as well as correlation with fat mass (FM) and fat-free mass (FFM) estimations, when compared with reference methods [5]. The surveyors note that the reproducibility is due to easy handling of the devices and there was little variation due to the handler. The majority of the studies in the survey were single-frequency studies; however, 5 of the 30 studies used multi-frequency BIA (MF-BIA) and BIS. Whole body BIA is not sensitive to fluid changes in the trunk, which segmental BIA addresses [6]. Additionally, whole body BIA assumes the individual regions of the body do not significantly differ in composition, which is not necessarily the case [7]. The relative limb lengths of some subjects may differ from the population used for population equations, which whole-body BIA may not address [8]. One study found that, on an individual basis, whole-body BIS did not significantly improve on anthropometry alone [9]. The authors of that study only noted slight improvements and believe that some added information about compartments or segments, such as lengths or diameters, may improve predictions. Another study compared whole-body and segmental BIS to evaluate the body composition of subject with a pitting edema in one leg, before and after a surgery [10]. In that study, whole-body BIA was performed twice, once for the right side and once for the left side of the body, and the authors found that whole-body BIA was appropriate for overall body composition only when measuring on the side opposite the affected leg. Whole-body BIA tends to underestimate fat mass in the trunk, and a potential reason based on trunk circumference was explored [11]. The authors note that in those who are overweight, soft tissue hydration is significantly different. In addition, variations in body shape from the population the prediction equation is based on will affect the result.

\subsection{Segmental BIA Background}

Segmental bioelectric impedance analysis provides an alternative to the BIA methods that analyze the body as a whole. Instead of modelling the whole body as a single cylinder in prediction equations, individual segments are modeled independently, such as the arms, trunk, and legs; however, a variety of other methods using more segments or focusing on certain segments have also been explored $[6,8]$. There are a variety of methods for placing electrodes for segmental measurements. The current electrodes may be moved to the segment, though it was noted that the sum of the segment measurements was greater than the corresponding whole-body measurement [12]. Segmental measurements may be achieved by placing electrodes at both sides, at each hand and foot, instead of just on one side, as is the case in whole-body measurements [7]. Another option is placing voltage sensing electrodes at the shoulder and upper anterior iliac spine, in addition to the wrist and ankle electrodes $[7,13]$. Some scales are available that measure foot-to-foot impedance while measuring body weight, providing body composition measurements [12]. Additional devices that provide measurements while the subject is standing have hand grips and foot pads that allow for segmental measurements [14]. These devices provide significantly larger impedance measurements to the more traditional method with wrist and ankle electrodes. This is because these methods include measurement of the hands, wrists, feet, and ankles, while the traditional method starts after the boniest parts of the 
wrist and ankle. The wrist and ankle combined can have resistances over half that of the whole-body resistance, despite contributing only around $1.8 \%$ to the body weight [15].

\subsection{Purpose}

Segmental BIS appears to be a suitable method for keeping the positives of BIA, while addressing the issues with sensitivity to changes in the trunk found in whole-body analysis. The purpose of this preliminary study was to validate the design of a new segmental BIS device (SBISD) capable of measuring the whole body, as well as the individual arm, trunk, and leg segments. The impedance measurements from the SBISD were directly compared against three existing whole-body BIS devices, including the Impedimed SFB7, the gold standard for whole-body BIS devices. The body resistances $\mathrm{R}_{0}$ and $R_{\infty}$ determined with the SBISD should be within $10 \%$ of a reference bioelectrical impedance analyzer.

\section{Material and Methods}

\subsection{Materials}

The test equipment and prototype device, referred to as the SBISD from now on, are presented in Figure 1. A signal generator (1) was used to supply a $1 \mathrm{~V}_{\mathrm{pp}}$ sine wave. A benchtop power supply (2) was used to power the op-amps. A digital oscilloscope (3) was used to measure the peak-to-peak voltage of two signals and the phase difference of the SBISD (4) outputs.

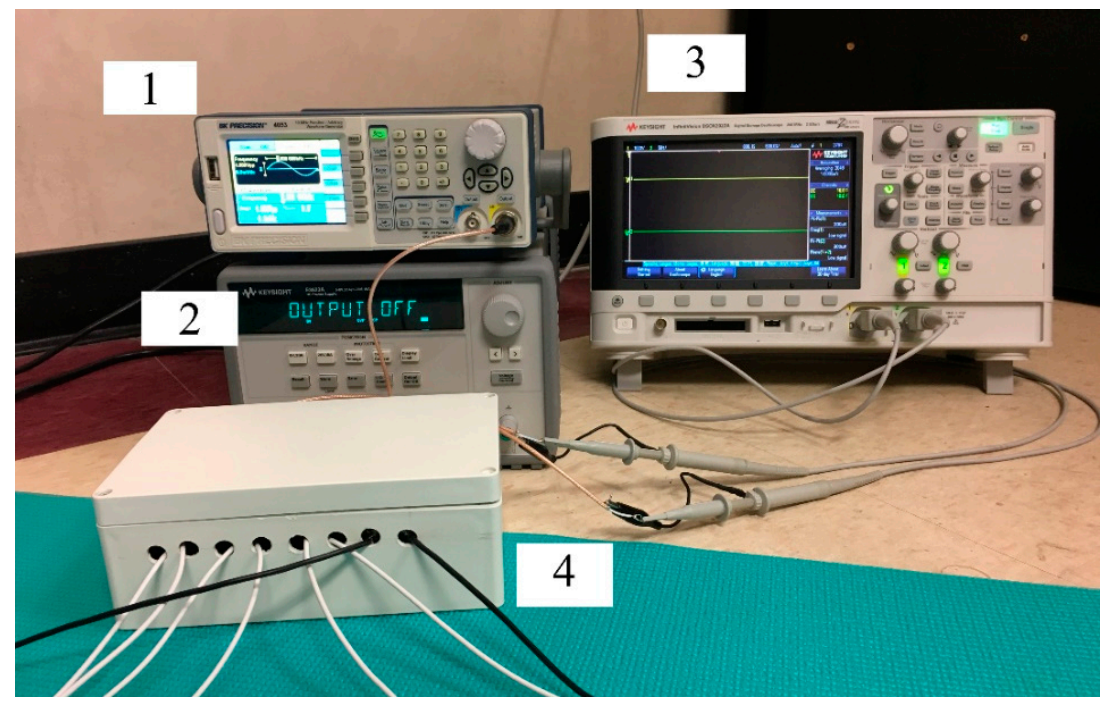

Figure 1. Test equipment and the segmental bioelectrical impedance spectroscopy device (SBISD): (1) signal generator, (2) power supply, (3) digital oscilloscope, and (4) SBISD.

Body composition measurements were performed with a Bodystat Multiscan 5000 (Bodystat Ltd., Douglas, Isle of Man), the Seca 514 Medical Body Composition Analyzer (seca GmbH \& Co. KG., Hamburg, Germany), and SFB7 (Impedimed Ltd., Pinkenba, QLD, Australia).

\subsection{SBISD Prototype for Whole-Body Measurement}

An initial prototype of the SBISD was constructed for whole-body measurement so that it could be validated. For prototype testing, a signal generator was used to provide a $1 \mathrm{~V}_{\mathrm{pp}}$ sine wave from $3 \mathrm{kHz}$ to $1 \mathrm{MHz}$. This signal was input to the current pump, which was designed to provide a constant current of $280 \mu \mathrm{A}_{\mathrm{pp}}$ given a $1 \mathrm{~V}_{\mathrm{pp}}$ input. The ends of the current pump were attached to reference

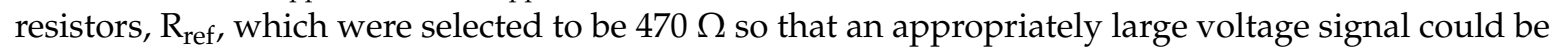
measured. Then, leads were attached to the other ends of the reference resistors. These leads connected to the object under test, $Z_{\text {test }}$, for injecting current. Multiple instrumentation amplifier (IA) circuits were 
constructed so that the signal would be amplified to twice the magnitude of the original. The first, IA1, measured the voltage across $R_{\text {ref }}$ for a reference measurement. The second, IA2, had leads attached and measured the voltage across $Z_{\text {test }}$. The outputs of the IAs was connected to the oscilloscope, which was used to measure the peak-to-peak voltage of each output and the phase difference between the two signals. Two more instrumentation amplifiers were added so that segmental measurements could be performed. The block diagram is presented in Figure 2. With these additions, $Z_{\text {test }}$ was separated into three separate segments, allowing for individual measurement of the arm, trunk, and leg in humans.

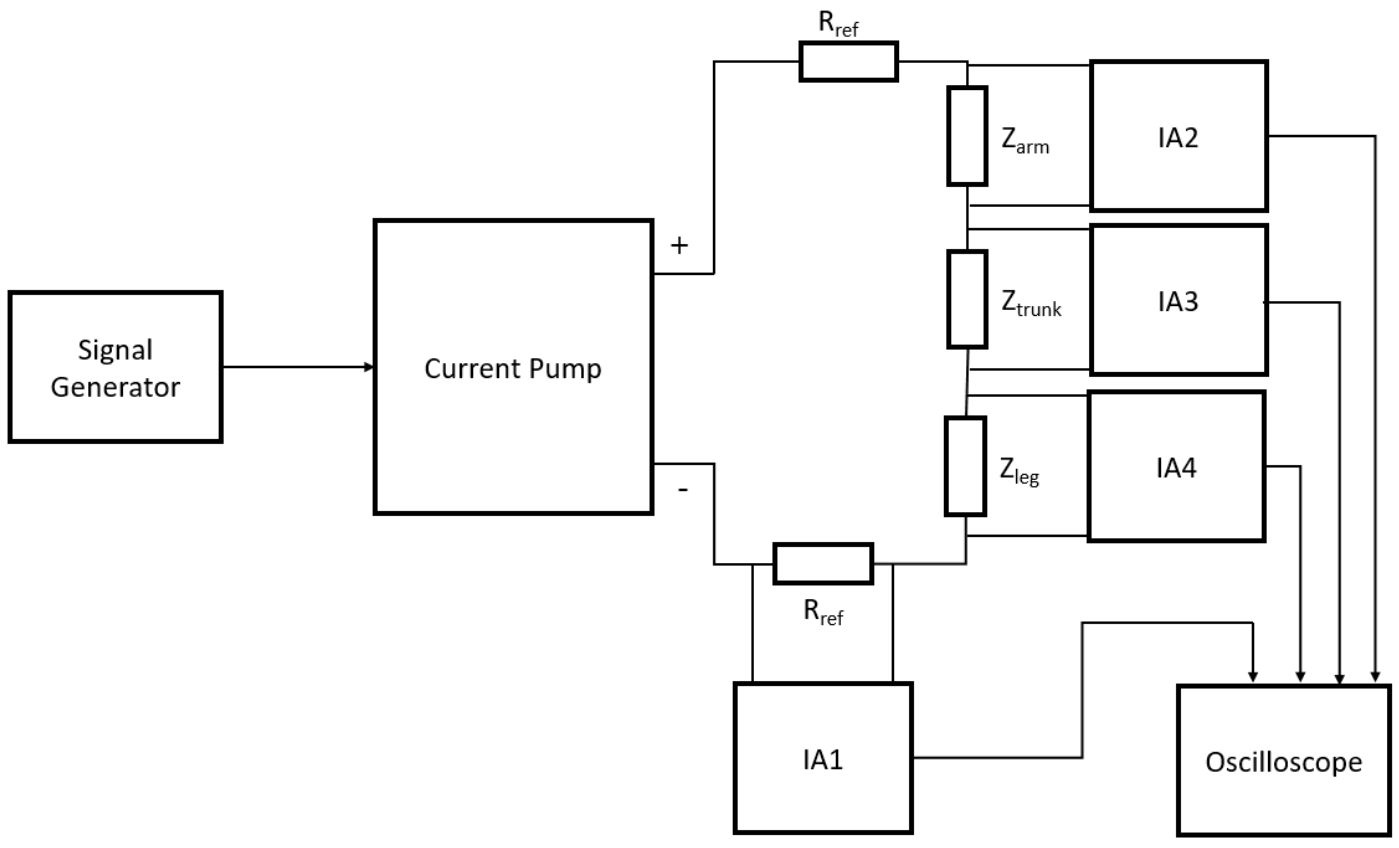

Figure 2. Block diagram for segmental measurements. IA-instrumentation amplifier.

\subsection{Validation with Existing Devices}

After completion of the prototype SBISD, a trial in humans was approved by the Georgia Southern University Institutional Review Board. Subjects volunteered and then signed a consent form to participate and allow for the sharing of data. The five subjects had a mean body mass of $68.8 \mathrm{~kg}$ with a standard deviation of $7.9 \mathrm{~kg}$. The mean height was $178 \mathrm{~cm}$ with a standard deviation of $5.3 \mathrm{~cm}$. The mean age of the subjects was 24 years, with a standard deviation of 4 years.

Along with the SBISD, several existing BIA devices were used for validating its impedance measurements. The first device used was the Seca 514. The next device used was the Bodystat Multiscan 5000. The third commercial device used was the Impedimed SFB7. It also required the subject lay supine. Finally, the SBISD was used. Measurements were first performed with the Seca 514. The Seca 514 has a built-in scale, with which subject body mass was recorded. This scale has an accuracy of $\pm 0.3 \%$ in the range of $35 \mathrm{~kg}$ to $300 \mathrm{~kg}$ [16]. Subject height was entered and then a BIA measurement was taken. The raw impedance data provided by the device was recorded. Next, the Multiscan 5000 was used to measure whole-body impedance. Gel tab electrodes were placed at the hand and foot following the guidelines from Bodystat, and the same height and body mass were entered as were used for the Seca 514 measurement. The subject lay supine for $5 \mathrm{~min}$ and then the measurement was performed. Then, the SFB7 was used to perform the same measurements, with the same electrodes as the Multiscan 5000 and the same height and body mass as used in the Seca 514 measurement. Finally, the SBISD was used to measure whole-body impedance with the same electrodes as the Multiscan 5000 and SFB7. The average impedance for each value was plotted in MATLAB (MathWorks, Natick, MA, USA), then a circle fit was performed. The resistances where this circle crossed the $x$-axis, $\mathrm{R}_{0}$, and $R_{\infty}$, were recorded. 


\subsection{Segmental Validation}

The SBISD was compared with the Seca 514, Multiscan 5000, and SFB7 for segmental measurements. The Seca 514 reports segment data for each arm and leg, as well as the right and left side of the body and the torso. Since all of these were reported at the same time, the segmental data from the whole-body procedure was used. Additional electrodes were placed for measuring individual body segments, including the arm, trunk, and leg. These electrode placements are presented in Figure 3 . Voltage sensing electrodes were added at the shoulder and waist, at locations 4 and 7, respectively. The remaining electrodes added-electrodes 3, 5, 6, and 8-were used for current injection, as the leads of the Multiscan 5000 did not allow for being left at the hand and foot for all measurements. Segmental measurements were recorded three times for each segment with the Multiscan 5000.

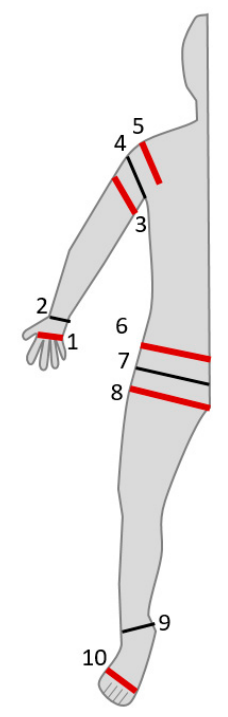

Figure 3. Electrode placements for segmental measurements.

\section{Results}

\section{Measuring Subjects 1 to 5}

The SBISD was used to measure the arm, leg, trunk, and whole body. The first set of leads was attached to the arm, the second to the trunk, and the third to the leg. Then, the second set of leads was used to measure the whole body. Each measurement was repeated for a total of three measurements. The average resistance and reactance values of subject 1 are presented in Figures 4-7, with error bars showing the spread of the measurements. The whole body, presented in Figure 4, was determined to have an $R_{0}$ of $647.6 \Omega$ and an $R_{\infty}$ of $441.2 \Omega$. The standard error of the estimate (SEE) for the whole body was calculated to be $1.0 \Omega$.

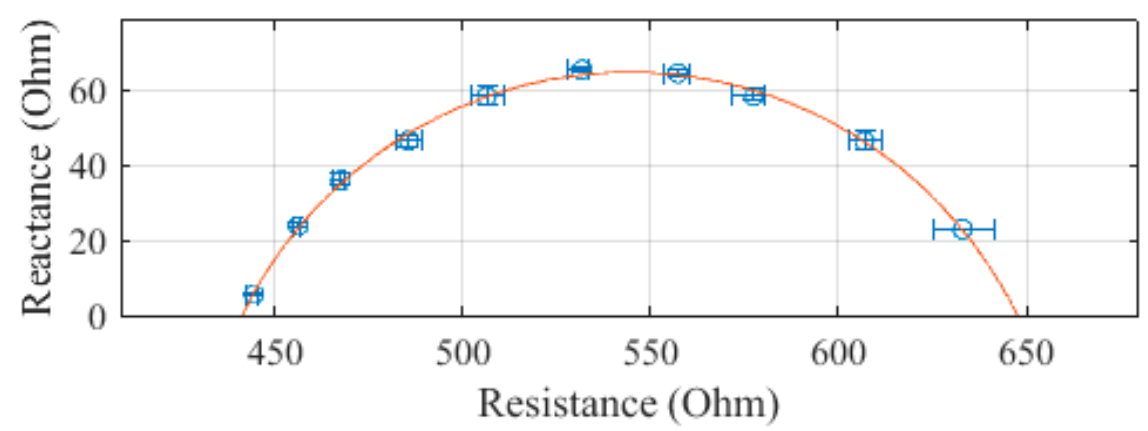

Figure 4. Cole-Cole plot of the whole-body impedance of subject 1 measured with the SBISD. 
The arm, plotted in Figure 5, was measured to have an $R_{0}$ of $334.4 \Omega$ and an $R_{\infty}$ of $219.2 \Omega$. The SEE was calculated to be $1.6 \Omega$ for the arm segment.

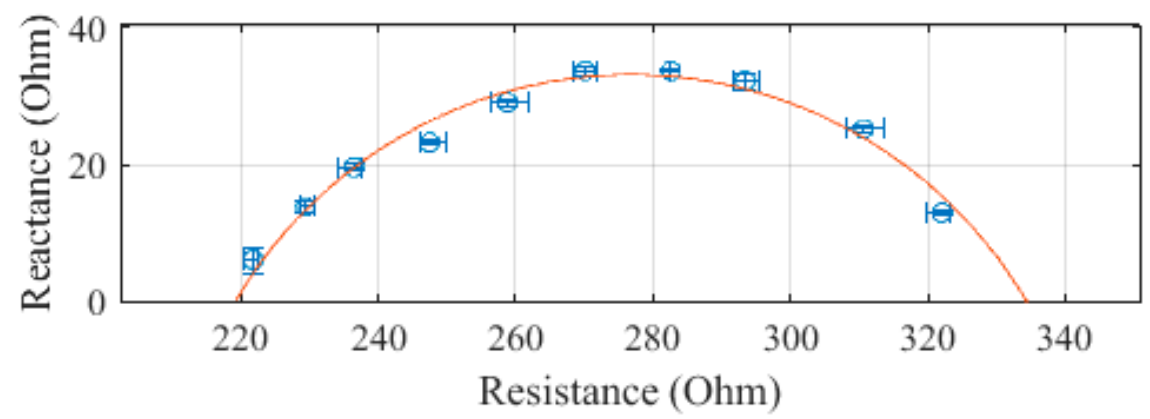

Figure 5. Cole-Cole plot of the arm impedance of subject 1 measured with the SBISD.

The trunk, plotted in Figure 6, was measured to have an $R_{0}$ of $75.3 \Omega$ and an $R_{\infty}$ of $42.3 \Omega$. The SEE was calculated to be $0.4 \Omega$ for the trunk segment.

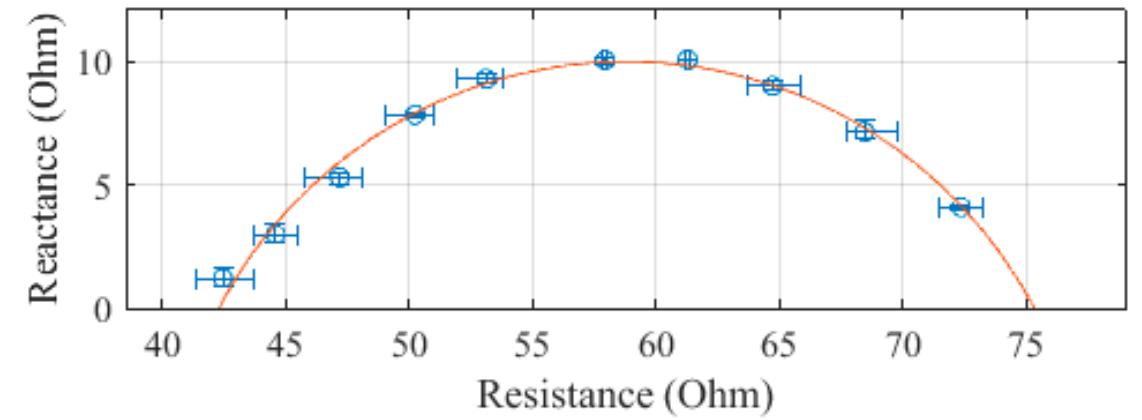

Figure 6. Cole-Cole plot of the trunk impedance of subject 1 measured with the SBISD.

The leg, plotted in Figure 7, was measured to have a $R_{0}$ of $333.9 \Omega$ and an $R_{\infty}$ of $216.5 \Omega$. The SEE was calculated to be $0.7 \Omega$ for the leg segment.

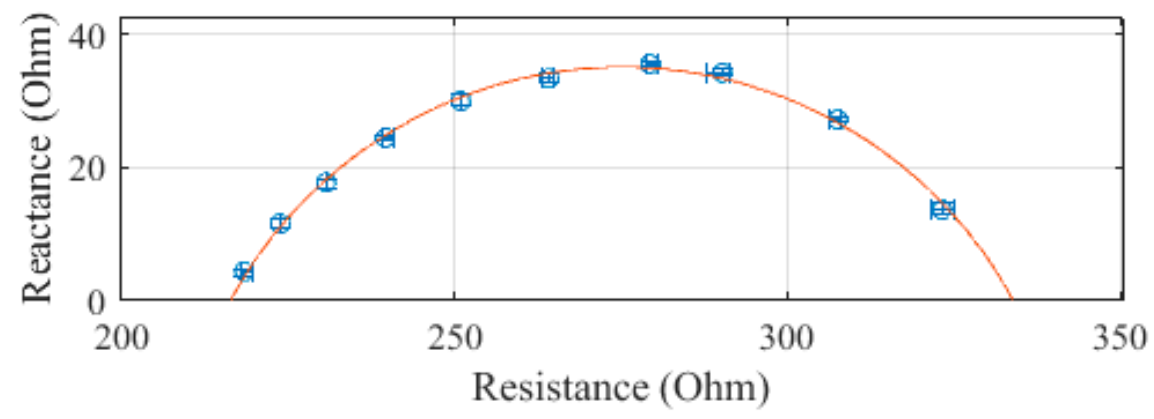

Figure 7. Cole-Cole plot of the leg impedance of subject 1 measured with the SBISD.

The same segments were measured with the Multiscan 5000, with each measurement repeated three times. The Cole-Cole plots of these measurements on subject 1 are presented in Figures 8-11. The average of the three measurements was plotted, with error bars to show the spread of the measurements. The whole body, plotted in Figure 8, was determined to have an $R_{0}$ of $662.0 \Omega$ and an $R_{\infty}$ of $455.8 \Omega$. There was an SEE of $1.2 \Omega$ for the whole body. 


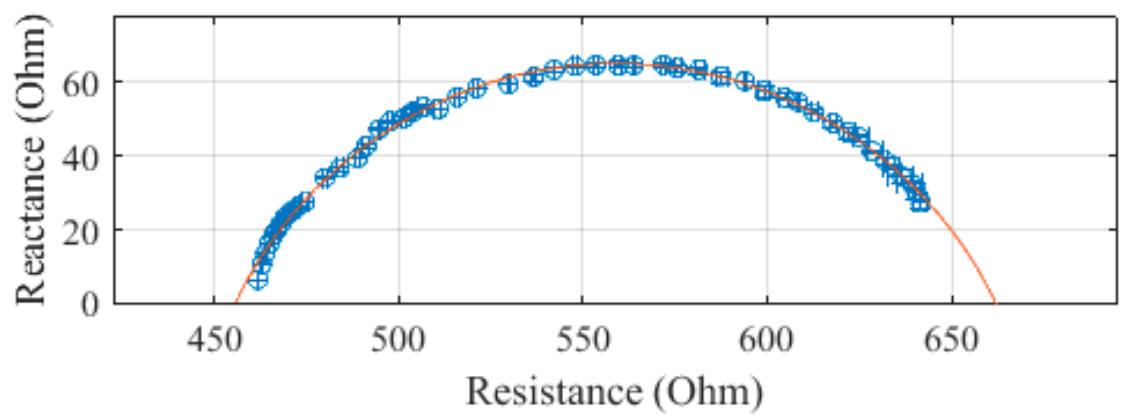

Figure 8. Cole-Cole plot of the whole-body impedance of subject 1 measured with a Multiscan 5000.

The arm was measured to have an $R_{0}$ of $335.7 \Omega$ and an $R_{\infty}$ of $230.5 \Omega$. The high frequencies deviated from the fit circle, leading to an SEE of $1.5 \Omega$.

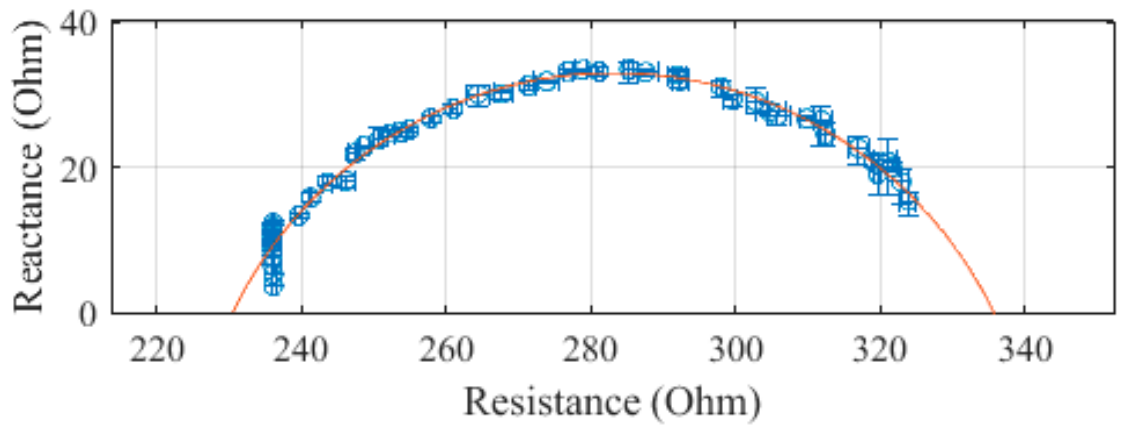

Figure 9. Cole-Cole plot of the arm impedance of subject 1 measured with a Multiscan 5000.

The trunk was measured to have an $R_{0}$ of $80.1 \Omega$ and an $R_{\infty}$ of $36.9 \Omega$. The SEE for the trunk segment was calculated to be $0.7 \Omega$.

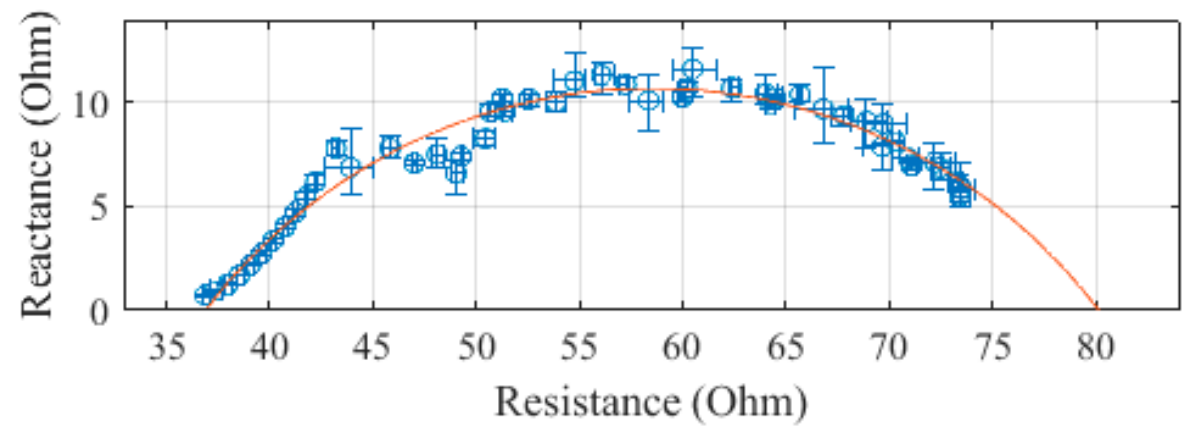

Figure 10. Cole-Cole plot of the trunk impedance of subject 1 measured with a Multiscan 5000.

The leg was measured to have an $R_{0}$ of $348.3 \Omega$ and an $R_{\infty}$ of $229.0 \Omega$. The leg segment was calculated to have an SEE of $1.7 \Omega$. 


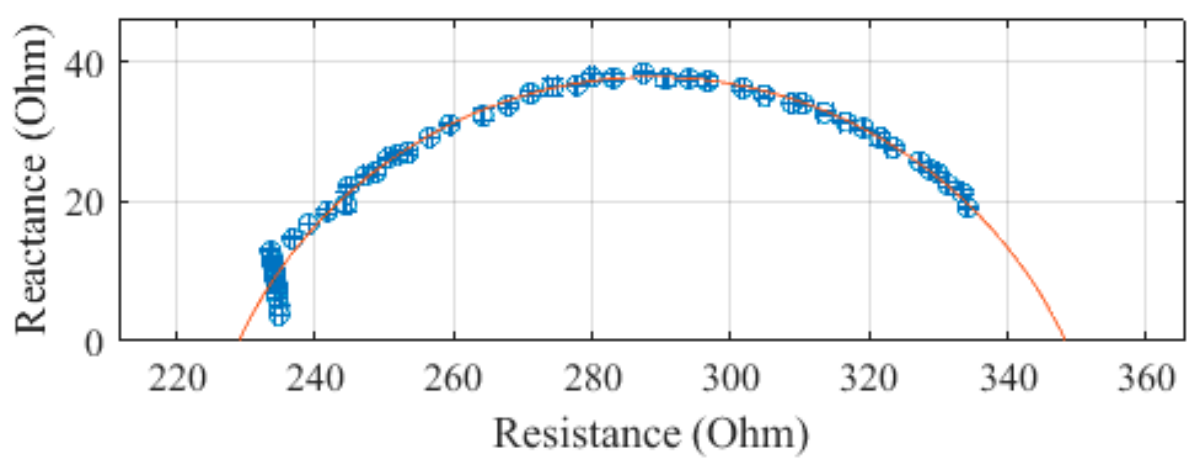

Figure 11. Cole-Cole plot of the leg impedance of subject 1 measured with a Multiscan 5000.

The second device used at this stage was the Seca 514. This device provides the resistance and reactance values for the whole body and each segment after each measurement. The arm and leg segments were compensated to account for the hook effect in Excel 2016 (Microsoft Corp., Redmond, WA, USA), and the measurements below $3 \mathrm{kHz}$ were discarded. The Cole-Cole plots of the average measurement for each segment of subject 1 are presented in Figures 12-15. The whole body was determined to have an $R_{0}$ of $795.0 \Omega$ and an $R_{\infty}$ of $575.0 \Omega$. The SEE of the whole-body measurement was calculated to be $0.9 \Omega$.

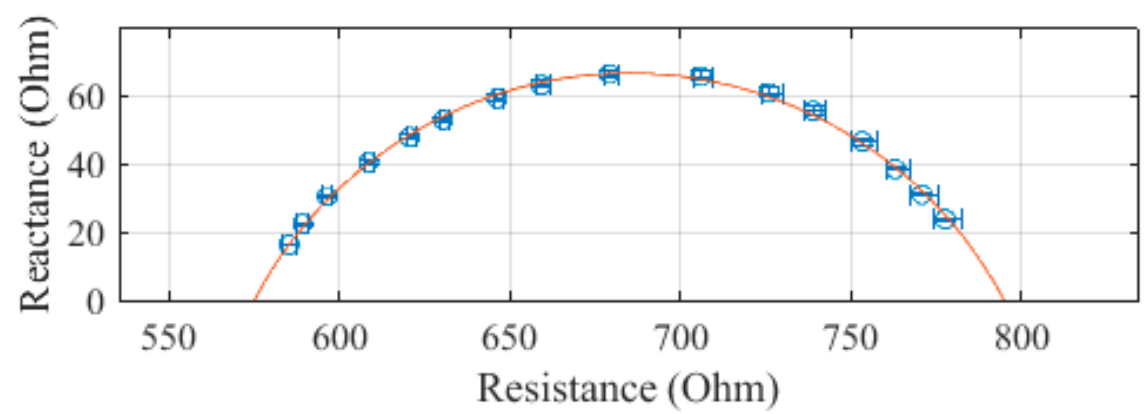

Figure 12. Cole-Cole plot of the whole-body impedance of subject 1 measured with a Seca 514 .

The arm was measured to have an $R_{0}$ of $461.6 \Omega$ and an $R_{\infty}$ of $330.2 \Omega$. The SEE was calculated to be $1.0 \Omega$ for the arm segment.

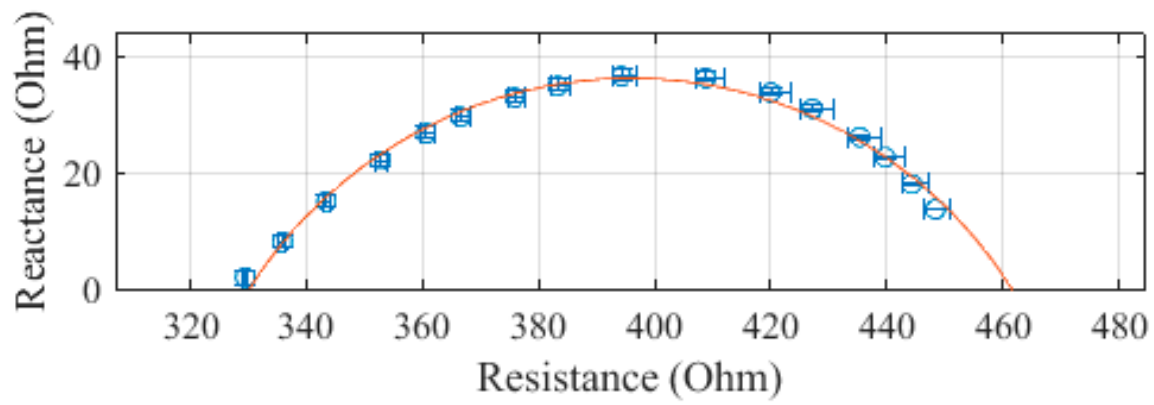

Figure 13. Cole-Cole plot of the arm impedance of subject 1 measured with a Seca 514.

For the torso, measurements above $300 \mathrm{kHz}$ were discarded due to large errors. The trunk was measured to have an $R_{0}$ of $30.0 \Omega$ and an $R_{\infty}$ of $21.3 \Omega$. The SEE, after removing the points, was calculated to be $0.01 \Omega$. 


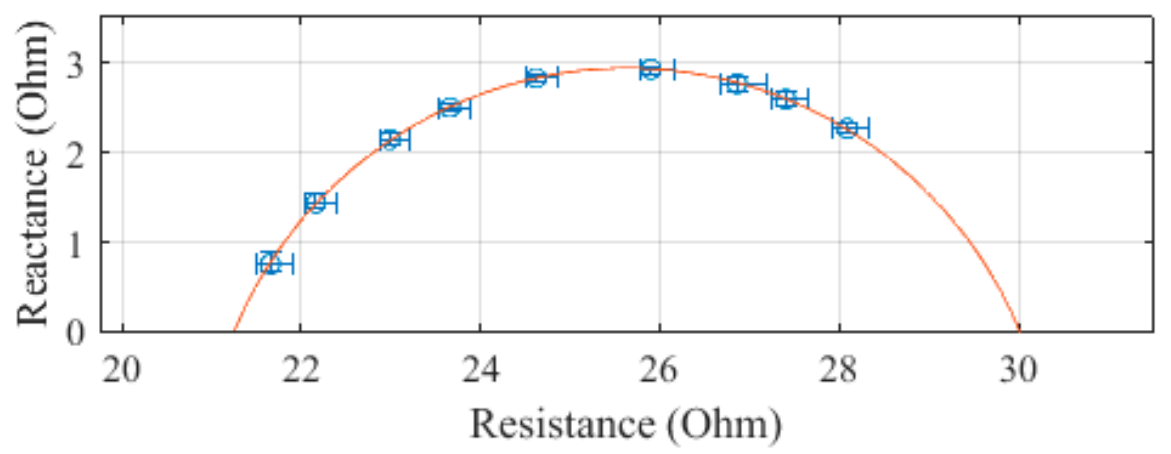

Figure 14. Cole-Cole plot of the trunk impedance of subject 1 measured with a Seca 514.

The leg was measured to have an $R_{0}$ of $303.7 \Omega$ and an $R_{\infty}$ of $219.9 \Omega$. The SEE for the leg segment was calculated to be $0.6 \Omega$.

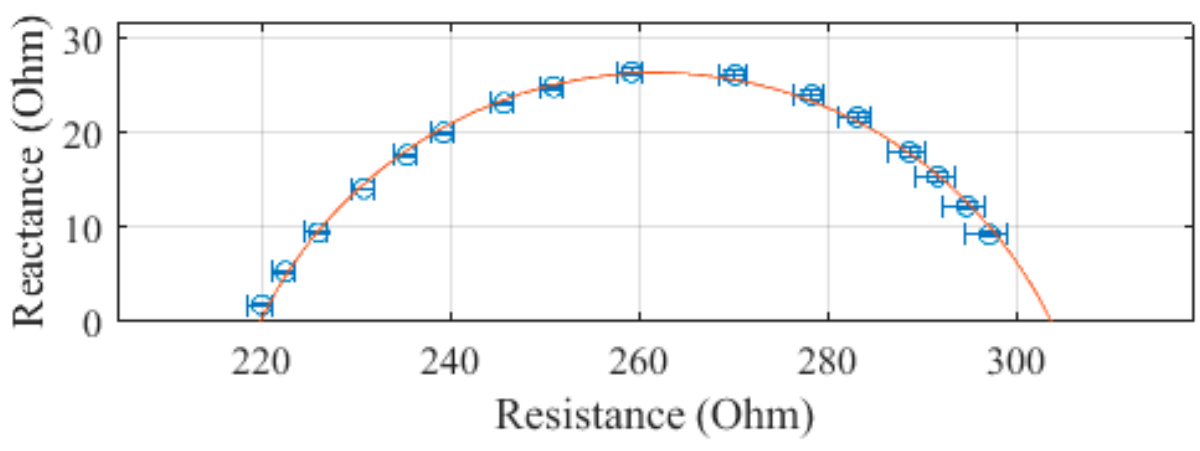

Figure 15. Cole-Cole plot of the leg impedance of subject 1 measured with a Seca 514.

Finally, the whole body and each segment were also measured with the SFB7. The Cole-Cole plots for each segment of subject 1 are presented in Figures 16-19. Each data point is the average impedance measured at a frequency, with the error bars indicating the spread of the data. The SFB7 software marked data points to be discarded, so these data points were discarded before plotting, reducing the data to the range of $5 \mathrm{kHz}$ to $500 \mathrm{kHz}$. In this frequency range, $R_{0}$ was found to be $657.7 \Omega, R_{\infty}$ was found to be $458.6 \Omega$, and the SEE was calculated to be $0.6 \Omega$ for the whole-body measurement.

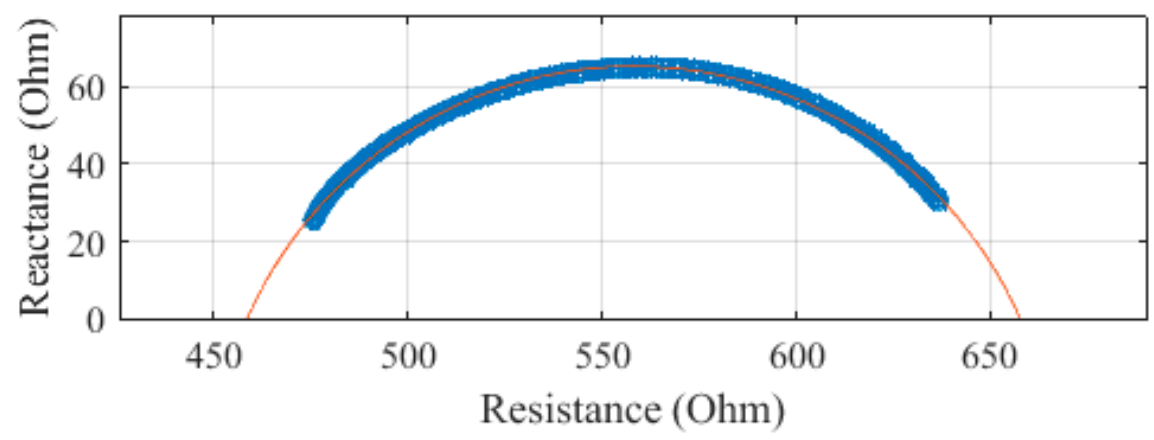

Figure 16. Cole-Cole plot of the whole-body impedance of subject 1 measured with a SFB7.

The arm was measured to have an $R_{0}$ of $331.2 \Omega$ and an $R_{\infty}$ of $228.3 \Omega$. The SEE for the arm segment measurements was calculated to be $0.4 \Omega$. 


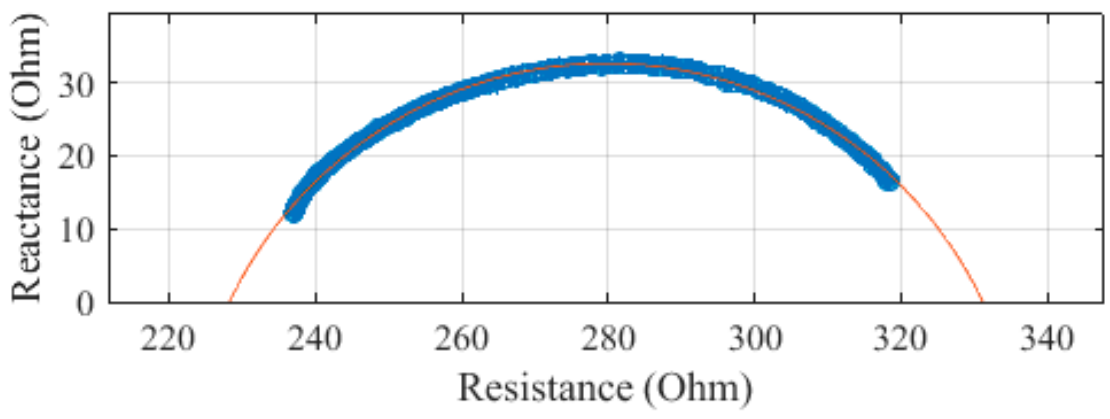

Figure 17. Cole-Cole plot of the arm impedance of subject 1 measured with a SFB7.

The trunk was measured to have an $R_{0}$ of $77.9 \Omega$ and an $R_{\infty}$ of $41.2 \Omega$. The SEE for the trunk segment was found to be $0.1 \Omega$.

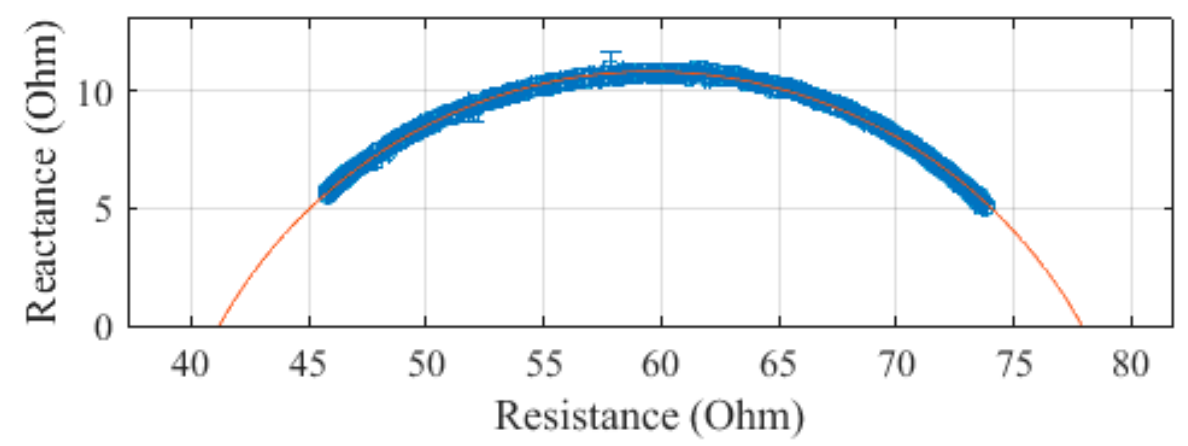

Figure 18. Cole-Cole plot of the trunk impedance of subject 1 measured with a SFB7.

The leg was measured to have an $R_{0}$ of $348.6 \Omega$ and an $R_{\infty}$ of $231.5 \Omega$. The SEE of the leg segment was calculated to be $0.3 \Omega$.

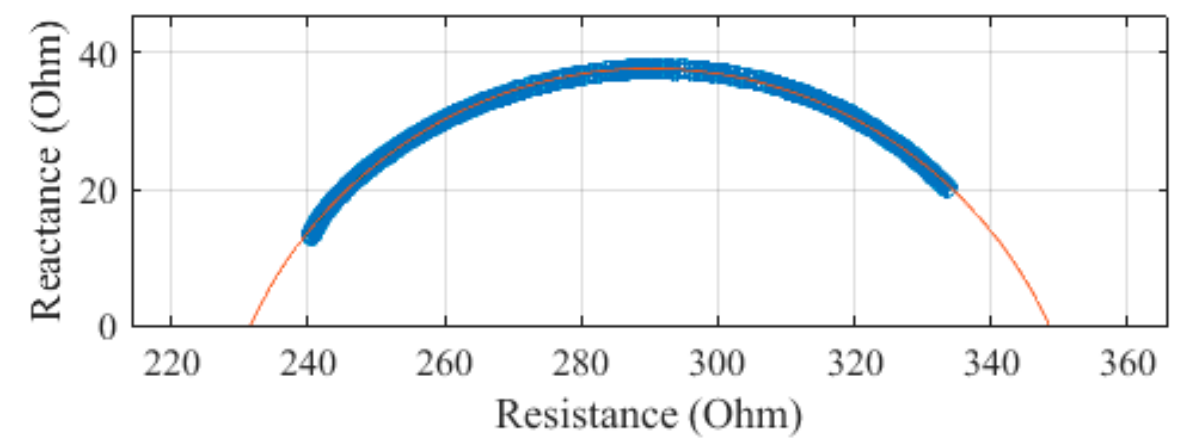

Figure 19. Cole-Cole plot of the leg impedance of subject 1 measured with a SFB7.

A comparison of the $R_{0}$ and $R_{\infty}$ values determined with the three devices is presented in Table 1 . 
Table 1. Device comparison for subject 1.

\begin{tabular}{cccccc}
\hline \multirow{2}{*}{ Whole body } & & SBISD & MS5000 & Seca 514 & SFB7 \\
\cline { 2 - 6 } & $\mathrm{R}_{0}$ & 647.6 & 662.0 & 795.0 & 657.7 \\
\cline { 2 - 6 } Arm & $\mathrm{R}_{\infty}$ & 441.2 & 455.8 & 575.0 & 458.6 \\
\cline { 2 - 6 } & $\mathrm{R}_{0}$ & 334.4 & 335.1 & 461.6 & 331.2 \\
\hline \multirow{2}{*}{ Trunk } & $\mathrm{R}_{\infty}$ & 219.2 & 230.5 & 330.2 & 228.3 \\
\cline { 2 - 6 } & $\mathrm{R}_{0}$ & 75.3 & 80.1 & 30.0 & 77.9 \\
\hline \multirow{2}{*}{ Leg } & $\mathrm{R}_{\infty}$ & 42.3 & 36.9 & 21.3 & 41.2 \\
\cline { 2 - 6 } & $\mathrm{R}_{0}$ & 333.9 & 348.3 & 303.7 & 348.6 \\
\hline
\end{tabular}

This procedure was repeated with subjects $2-5$ for whole-body and segmental measurements. A comparison of the SBISD segmental measurements to the three reference devices, for all five subjects, is presented in Table 2. Each cell shows the percent difference of the SBISD to the reference devices. The largest differences were found when compared with the Seca 514, since the Seca 514 measures from the fingers to the heel, as opposed to wrist to ankle, measuring the bony wrist, hand, and ankle [15].

Table 2. Segmental measurement differences versus SBISD measure.

\begin{tabular}{|c|c|c|c|c|c|}
\hline & & & \multicolumn{3}{|c|}{$\%$ Difference } \\
\hline & & & MS5000 & Seca 514 & SFB7 \\
\hline \multirow{8}{*}{ Subject 1} & \multirow{2}{*}{ Whole-Body } & $\mathrm{R}_{0}$ & -2.2 & -18.5 & -1.5 \\
\hline & & $\mathrm{R}_{\infty}$ & -3.2 & -23.3 & -3.8 \\
\hline & \multirow{2}{*}{ Arm } & $\mathrm{R}_{0}$ & -0.2 & -27.6 & 1.0 \\
\hline & & $\mathrm{R}_{\infty}$ & -4.9 & -33.6 & -4.0 \\
\hline & \multirow{2}{*}{ Trunk } & $\mathrm{R}_{0}$ & -6.0 & 151.0 & -3.3 \\
\hline & & $\mathrm{R}_{\infty}$ & 14.6 & 98.6 & 2.7 \\
\hline & \multirow{2}{*}{ Leg } & $\mathrm{R}_{0}$ & -4.1 & 9.9 & -4.2 \\
\hline & & $\mathrm{R}_{\infty}$ & -5.5 & -1.5 & -6.5 \\
\hline \multirow{8}{*}{ Subject 2} & \multirow{2}{*}{ Whole-Body } & $\mathrm{R}_{0}$ & -2.2 & -20.9 & -1.7 \\
\hline & & $\mathrm{R}_{\infty}$ & -5.0 & -27.3 & -6.8 \\
\hline & \multirow{2}{*}{ Arm } & $\mathrm{R}_{0}$ & -3.0 & -33.6 & -1.9 \\
\hline & & $\mathrm{R}_{\infty}$ & -3.2 & -35.4 & -5.7 \\
\hline & \multirow{2}{*}{ Trunk } & $\mathrm{R}_{0}$ & -6.6 & 119.5 & -4.4 \\
\hline & & $\mathrm{R}_{\infty}$ & 9.3 & 68.7 & -19.4 \\
\hline & \multirow{2}{*}{ Leg } & $\mathrm{R}_{0}$ & -3.4 & 12.3 & -1.8 \\
\hline & & $\mathrm{R}_{\infty}$ & -4.0 & -3.7 & -7.9 \\
\hline \multirow{8}{*}{ Subject 3} & \multirow{2}{*}{ Whole-Body } & $\mathrm{R}_{0}$ & -1.4 & -16.6 & -1.5 \\
\hline & & $\mathrm{R}_{\infty}$ & -0.7 & -20.6 & -2.9 \\
\hline & \multirow{2}{*}{ Arm } & $\mathrm{R}_{0}$ & -3.0 & -29.1 & -1.2 \\
\hline & & $\mathrm{R}_{\infty}$ & -1.9 & -32.5 & -7.9 \\
\hline & \multirow{2}{*}{ Trunk } & $\mathrm{R}_{0}$ & -8.1 & 187.0 & -2.4 \\
\hline & & $\mathrm{R}_{\infty}$ & 9.0 & 106.2 & -23.5 \\
\hline & \multirow{2}{*}{ Leg } & $\mathrm{R}_{0}$ & -3.4 & 12.3 & -2.3 \\
\hline & & $\mathrm{R}_{\infty}$ & -0.8 & -1.0 & -3.9 \\
\hline
\end{tabular}


Table 2. Cont.

\begin{tabular}{|c|c|c|c|c|c|}
\hline & & & \multicolumn{3}{|c|}{ \% Difference } \\
\hline & & & MS5000 & Seca 514 & SFB7 \\
\hline \multirow{8}{*}{ Subject 4} & \multirow{2}{*}{ Whole-Body } & $\mathrm{R}_{0}$ & -0.8 & -15.9 & -2.2 \\
\hline & & $\mathrm{R}_{\infty}$ & -3.1 & -21.8 & -4.2 \\
\hline & \multirow{2}{*}{ Arm } & $\mathrm{R}_{0}$ & -0.4 & -27.3 & -2.0 \\
\hline & & $\mathrm{R}_{\infty}$ & -4.7 & -30.8 & -7.3 \\
\hline & \multirow{2}{*}{ Trunk } & $\mathrm{R}_{0}$ & -4.2 & 188.2 & -3.1 \\
\hline & & $\mathrm{R}_{\infty}$ & 4.5 & 116.6 & 1.4 \\
\hline & \multirow{2}{*}{ Leg } & $\mathrm{R}_{0}$ & -1.9 & 12.0 & -0.8 \\
\hline & & $\mathrm{R}_{\infty}$ & -3.4 & -1.4 & -3.5 \\
\hline \multirow{8}{*}{ Subject 5} & \multirow{2}{*}{ Whole-Body } & $\mathrm{R}_{0}$ & -0.6 & -16.1 & -0.6 \\
\hline & & $R_{\infty}$ & -1.6 & -20.9 & -1.4 \\
\hline & \multirow{2}{*}{ Arm } & $\mathrm{R}_{0}$ & -1.2 & -28.3 & -1.3 \\
\hline & & $\mathrm{R}_{\infty}$ & -2.3 & -31.9 & -0.9 \\
\hline & \multirow{2}{*}{ Trunk } & $\mathrm{R}_{0}$ & -6.8 & 158.8 & -3.7 \\
\hline & & $\mathrm{R}_{\infty}$ & 6.8 & 105.9 & 6.1 \\
\hline & \multirow{2}{*}{ Leg } & $\mathrm{R}_{0}$ & -0.8 & 20.1 & -0.4 \\
\hline & & $\mathrm{R}_{\infty}$ & -0.1 & 7.4 & -0.3 \\
\hline
\end{tabular}

While the $R_{0}$ and $R_{\infty}$ values may differ, it was investigated whether the width of the Cole-Cole curve was similar for the devices. These values are presented in Table 3. The segments as defined in this experiment likely differed from those defined by the Seca, which could contribute to the differences noted.

Table 3. Widths of the Cole-Cole Curves.

\begin{tabular}{|c|c|c|c|c|c|}
\hline & & \multicolumn{4}{|c|}{$\mathbf{R}_{0}-\mathbf{R}_{\infty}(\Omega)$} \\
\hline & & SBISD & MS5000 & Seca 514 & SFB7 \\
\hline \multirow{4}{*}{ Subject 1} & Whole Body & 206.4 & 206.2 & 220.0 & 199.1 \\
\hline & Arm & 115.2 & 104.6 & 131.4 & 102.9 \\
\hline & Trunk & 33.0 & 43.2 & 8.7 & 36.7 \\
\hline & Leg & 117.4 & 119.3 & 83.8 & 117.1 \\
\hline \multirow{4}{*}{ Subject 2} & Whole Body & 220.0 & 213.4 & 235.6 & 202.5 \\
\hline & Arm & 93.9 & 96.6 & 133.4 & 88.1 \\
\hline & Trunk & 38.9 & 47.3 & 12.7 & 33.6 \\
\hline & Leg & 144.0 & 147.9 & 98.4 & 132.9 \\
\hline \multirow{4}{*}{ Subject 3} & Whole Body & 211.2 & 217.2 & 229.4 & 208.5 \\
\hline & Arm & 95.3 & 100.4 & 121.6 & 83.3 \\
\hline & Trunk & 47.3 & 57.7 & 11.5 & 38.2 \\
\hline & Leg & 132.5 & 142.9 & 92.9 & 131.9 \\
\hline \multirow{4}{*}{ Subject 4} & Whole Body & 230.5 & 219.7 & 227.0 & 224.7 \\
\hline & Arm & 104.3 & 93.5 & 126.4 & 91.9 \\
\hline & Trunk & 36.0 & 41.4 & 7.4 & 39.2 \\
\hline & Leg & 156.6 & 155.5 & 106.3 & 149.9 \\
\hline \multirow{4}{*}{ Subject 5} & Whole Body & 148.4 & 145.7 & 152.1 & 146.5 \\
\hline & Arm & 79.2 & 78.3 & 98.6 & 80.9 \\
\hline & Trunk & 31.4 & 38.0 & 9.0 & 35.6 \\
\hline & Leg & 80.2 & 82.2 & 47.5 & 80.7 \\
\hline
\end{tabular}


The commercial analyzers were then compared for their prediction of fat mass, fat-free mass, extracellular fluid, and intracellular fluid. These values are presented in Table 4.

Table 4. Comparison of Body Composition Parameters Determined by the Commercial Analyzers.

\begin{tabular}{ccccc}
\hline & & MS5000 & Seca 514 & SFB7 \\
\hline \multirow{5}{*}{ Subject 1 } & FM (kg) & 13.9 & 22.0 & 21.5 \\
& FFM (kg) & 66.5 & 58.4 & 58.9 \\
& ECF (L) & 17.7 & 17.0 & 19.2 \\
& ICF (L) & 23.8 & 24.9 & 23.9 \\
\hline \multirow{5}{*}{ Subject 2 } & FM (kg) & 5.7 & 9.1 & 8.2 \\
& FFM (kg) & 59.8 & 56.4 & 57.3 \\
& ECF (L) & 16.5 & 15.0 & 17.9 \\
& ICF (L) & 25.5 & 25.5 & 24.1 \\
\hline \multirow{5}{*}{ Subject 3 } & FM (kg) & 6.8 & 9.3 & 10.0 \\
& FFM (kg) & 63.8 & 60.5 & 60.5 \\
& ECF (L) & 17.4 & 17.0 & 18.6 \\
& ICF (L) & 27.1 & 26.8 & 25.7 \\
\hline & FM (kg) & 12.8 & 14.3 & 16.5 \\
& FFM (kg) & 46.0 & 44.5 & 42.3 \\
& ECF (L) & 13.0 & 13.5 & 12.9 \\
& ICF (L) & 16.9 & 18.7 & 18.0 \\
\hline \multirow{5}{*}{ Subject 5 } & FM (kg) & 3.9 & 6.1 & 4.6 \\
& FFM (kg) & 64.4 & 62.2 & 63.7 \\
& ECF (L) & 19.4 & 19.2 & 21.0 \\
& ICF (L) & 25.3 & 27.1 & 25.6 \\
\hline
\end{tabular}

\section{Discussion}

\subsection{Outcome}

The SBISD whole-body measurements were within 5\% of both the Multiscan 5000 and SFB7, except for $\mathrm{R}_{\infty}$ of subject 2 , where it differed by $5 \%$ from the Multiscan 5000 and $6.8 \%$ of the SFB7. For the arm and leg segments, all measurements with the SBISD were within $10 \%$ of those performed with the SFB7 and Multiscan 5000. For the trunk, several measurements at $R_{\infty}$ differed by more than $10 \%$ when compared with either the Multiscan 5000 or the SFB7. In the two cases of a trunk $R_{\infty}$ measurement differing by more than $10 \%$ from the SFB7, it was within $10 \%$ of the Multiscan 5000 . For the case where it was more than $10 \%$ from the Multiscan 5000 , it was within $10 \%$ of the SFB7. The $\mathrm{R}_{\infty}$ values for the trunk was in the $20-40 \Omega$ range, where a $10 \%$ error would be a $2-4 \Omega$ difference. Typically, the SBISD had a larger error when determining $R_{\infty}$ than $R_{0}$ when compared with the SFB7 or Multiscan 5000.

Trunk measurements led to the most discrepancies between the body composition analyzers. The trunk has a more complex makeup than the arm and leg in terms of the variety and layout of tissues [17]. The Multiscan 5000 and Seca 514 both showed increased error regarding measuring the trunk segments. For the Multiscan 5000, this resulted in less closeness to the circle fit and more spread in some data. The impedance values for the trunk were very close to the lower end of the measuring range of the Multiscan 5000, which was 20-1300 $\Omega$. For the Seca 514, this measuring error was evident due to much larger reactance values in the highest one to three frequencies depending on the subject. The measured trunk values were also very close to the low end of the Seca 514's impedance measuring range of 10-1000 $\Omega$. Measurement errors and insensitivity to fluid changes in the trunk has been discussed in many articles, so it is an area of interest for segmental measurement capability $[11,13,18,19]$. 


\subsection{How Segmental BIS Overcomes the Disadvantages of Whole-Body BIS}

In a review of segmental BIA, it was noted that a drawback of whole-body BIS is the different cross-section sizes of body parts, mainly the limbs versus the trunk [12]. For any given section with length $l$ and resistivity R, there will be a linear relationship between FFM and $l^{2} / \mathrm{R}$. However, for the whole body, this is not the case. Segmental BIA has been used to avoid this variation [7]. There are several conditions where whole-body measurement may not be valid but segmental measurement could be applicable, including for overweight individuals, detecting edemas for early diagnosis, and monitoring hydration in the trunk [7]. Since whole-body BIS is less sensitive to changes in the trunk, it may not be suitable for tracking changes in overweight individuals. A study sought to see whether segmental MF-BIA would be applicable using a standing device with hand grips and foot pads and measuring a variety of BMIs [14]. It was noted that the error compared to DXA increased as \%BF increased. For the normal and overweight groups, there was good agreement with a modest error. There was a significant overestimation of $\% \mathrm{BF}$ for BMIs that were in the obese category. In general, it underestimated \%BF for normal BMIs and overestimated it for overweight and obese BMIs. Segmental BIS can allow for tracking changes during recovery from disease, and how each limb is affected. The changes through recovery from dengue fever was monitored in one study [20]. They performed segmental and whole-body measurements on subjects that were either a control group ( 11 subjects) or a group of dengue fever patients (10 subjects), in the acute and recovery stages. Segmental measurements consisted of the arm, leg, and trunk, and BIS measurements were performed at 50 frequencies and consisted of ECF and ICF resistances. Segmental BIA can provide information about each limb individually, making it suitable for tracking changes in body composition, such as fat and muscle mass, and providing more information about the development of a child. A study aimed to collect data on how fat accumulated in Turkish children with ages between 7 and 18 to provide data for the future diagnosis and tracking of childhood obesity [21]. Measurements were performed on a total of 1371 subjects, measuring arm, leg, and trunk fat, as well as body fat and FFM. Anthropometry was used to determine BMI as a reference. The authors noted that the fat pattern in older children was more central, as opposed to being peripherally located in younger children. They also noted the differences in fat distribution for boys and girls. Whole-body BIA has struggled with the morbidly obese, which was addressed by one study that generated new prediction equations for segmental BIA [22]. The large FM is underestimated by whole-body BIA, while FFM is overestimated. Size and weight restrictions prevent usage of some reference methods, so a portable method is desirable. Segmental and whole-body measurements were taken with the same device at a frequency of $50 \mathrm{kHz}$, with DXA as the reference method. The FFM provided by the BIA device had a good correlation with that from DXA; however, it did overestimate FFM by $3.9 \mathrm{~kg}$, and this overestimation increased with larger amounts of FFM. For the segments, FM in the trunk was overestimated, while in the limbs, it was underestimated. The effect of the addition of proximal electrodes in segmental BIA was analyzed to see if it could overcome the issue of BIA overestimating \%BF in lean elderly and underestimating it in the obese elderly due to their body type differing from that used for prediction equations [23]. The study had 42 subjects and used dilution methods as the reference methods. Different electrode placements were analyzed, including whole body and several segmental models. They found that the addition of proximal electrodes helped to overcome the systematic bias in the \%BF predicted by the different electrode placement methods. This was achieved by adding the impedance ratio of proximal limb segments to distal segments.

\subsection{Comparability with Seca 514}

The Seca 514 provided significantly different impedance measurements compared to the other analyzers used, except in the case of some leg segments, particularly the $R_{\infty}$ value for the leg measurements. This is because it does not directly measure the segments in the same way. Instead of electrodes at the wrist and ankle for measuring voltage, it has foot pads that measure from the fingers to the heel. This means that it is measuring longer paths through the body, including the 
high-resistance ankles and wrists [15]. However, when looking at the range of the Cole-Cole curve on the resistance axis, the whole-body curves were generally in agreement, as shown in Table 3. In addition, when comparing the FM, FFM, ECF, and ICF measurements, they were generally within $10 \%$ of those found from the other commercial devices. The FM, FFM, ECF, and ICF values for each device are shown in Table 4. This shows that the Seca 514 could be used as a reference device for comparing these values with a tetrapolar BIA device, but not necessarily for comparing impedance measurements directly. In addition to the added wrist and ankle impedance, the differences in limb measurements for the Seca 514 could be explained in part by how it defines and measures the segments. Previous eight-electrode devices used six different current paths: right side of body, left side of body, hand to hand, foot to foot, left hand to right foot, and right hand to left foot. They then used these measurements to determine the impedance for each body segment [24]. For that device, arm and leg segments were defined from the foot to the halfway point in the trunk between the two arms or between the two legs. This means that some of the trunk was included in those measurements. The trunk measurement was determined by subtracting the right arm and right leg from the right side of the body measurement [18]. How the segment measurements were determined by the Seca 514 were not disclosed, but a similar method for trunk impedance must be used.

\section{Conclusions}

A segmental bioelectrical impedance spectroscopy device (SBISD) was designed and a prototype was implemented. The SBISD was then used to measure five subjects, and the results were compared against the results of three commercial devices. It was within 10\% for whole-body, arm, and leg measurements, and most trunk measurements when compared with two of the devices, the SFB7 and the Multiscan 5000. The third device used, the Seca 514, could not be compared directly for impedance measurements, but was used to compare the body composition (FM, FFM, ECF, and ICF) measurements of the other commercial devices. Compared to tetrapolar devices like the Multiscan 5000 and the SFB7, the SBISD has the added benefit of measuring three segments simultaneously due to the two additional measuring circuits. It is suggested that future work be done to address the issues in measuring the trunk. This might be addressed by increasing the number of current injection sites to increase the number of current paths so that the different areas with different makeups can be addressed. With the current measuring part of the SBISD validated, further components can be focused on, like signal generation and data acquisition, to create a standalone device.

Author Contributions: T.C. studied the method and carried out experiment and completed the writing of this paper. J.C. clarified the research content and ideas, analyzed the data, and edit and proofread the manuscript.

Funding: This research received no external funding.

Conflicts of Interest: The authors declare no conflict of interest.

\section{References}

1. Jaffrin, M.Y.; Morel, H. Body fluid volumes measurements by impedance: A review of bioimpedance spectroscopy (BIS) and bioimpedance analysis (BIA) methods. Med. Eng. Phys. 2008, 30, 1257-1269. [CrossRef]

2. Fosbøl, M.Ø.; Zerahn, B. Contemporary methods of body composition measurement. Clin. Physiol. Funct. Imaging 2015, 35, 81-97. [CrossRef] [PubMed]

3. Lingwood, B.E. Bioelectrical impedance analysis for assessment of fluid status and body composition in neonates-the good, the bad and the unknown. Eur. J. Clin. Nutr. 2013, 67 (Suppl. 1), S28-S33. [CrossRef] [PubMed] 
4. Lingwood, B.E.; Coghlan, J.P.; Ward, L.C.; Charles, B.G.; Colditz, P.B. Measurement of extracellular fluid volume in the neonate using multiple frequency bio-impedance analysis. Physiol. Meas. 2000, $21,251$. [CrossRef] [PubMed]

5. De Castro, J.A.; de Lima, T.R.; Silva, D.A. Body composition estimation in children and adolescents by bioelectrical impedance analysis: A systematic review. J. Bodyw. Mov. Ther. 2018, 22, 134-146. [CrossRef] [PubMed]

6. Ward, L.C. Segmental bioelectrical impedance analysis: An update. Curr. Opin. Clin. Nutr. Metab. Care 2012, 15, 424. [CrossRef] [PubMed]

7. Kyle, U.G.; Bosaeus, I.; De Lorenzo, A.D.; Deurenberg, P.; Elia, M.; Gómez, J.M.; Heitmann, B.L.; Kent-Smith, L.; Melchior, J.C.; Pirlich, M.; et al. Bioelectrical impedance analysis-Part I: Review of principles and methods. Clin. Nutr. 2004, 23, 1226. [CrossRef] [PubMed]

8. Turner, A.A.; Lozano-Nieto, A.; Bouffard, M. Comparison of segmental and global bioimpedance spectroscopy errors using generalizability theory. Physiol. Meas. 2002, 23, 43. [CrossRef]

9. Pichler, G.P.; Amouzadeh-Ghadikolai, O.; Leis, A.; Skrabal, F. A critical analysis of whole body bioimpedance spectroscopy (BIS) for the estimation of body compartments in health and disease. Med. Eng. Phys. 2013, 35, 616-625. [CrossRef]

10. Codognotto, M.; Piazza, M.; Frigatti, P.; Piccoli, A. Applied nutritional investigation: Influence of localized edema on whole-body and segmental bioelectrical impedance. Nutrition 2008, 24, 569-574. [CrossRef]

11. Haas, V.; Engeli, S.; Hofmann, T.; Riedl, A.; Haufe, S.; Kast, P.; Wiesner, S.; Böhnke, J.; Jordan, J.; Boschmann, M. Variations in truncal body circumferences affect fat mass quantification with bioimpedance analysis. Eur. J. Clin. Nutr. 2012, 66, 196-200. [CrossRef] [PubMed]

12. De Lorenzo, A.; Andreoli, A. Segmental bioelectrical impedance analysis. Curr. Opin. Clin. Nutr. Metab. Care 2003, 6, 551. [CrossRef]

13. Zhu, F.; Schneditz, D.; Levin, N.W. Estimation of trunk extracellular volume by bioimpedance. In Proceedings of the 20th Annual International Conference of the IEEE Engineering in Medicine and Biology Society, Hong Kong, China, 29 October-1 November 1998.

14. Shafer, K.J.; Siders, W.A.; Johnson, L.K.; Lukaski, H.C. Validity of segmental multiple-frequency bioelectrical impedance analysis to estimate body composition of adults across a range of body mass indexes. Nutrition 2009, 25, 25-32. [CrossRef] [PubMed]

15. Bosy-Westphal, A.; Jensen, B.; Braun, W.; Pourhassan, M.; Gallagher, D.; Müller, M.J. Quantification of whole-body and segmental skeletal muscle mass using phase-senitive 8-electrode medical bioelectrical impedance devices. Eur. J. Clin. Nutr. 2017, 71, 1061-1067. [CrossRef]

16. Seca. Seca 514-Instructions for Use; Seca Gmbh \& Co. KG: Hamburg, Germany, 2015.

17. Ishiguro, N.; Kanehisa, H.; Miyatani, M.; Masuo, Y.; Fukunaga, T. Applicability of segmental bioelectrical impedance analysis for predicting trunk skeletal muscle volume. J. Appl. Physiol. 2006, 100, 572. [CrossRef]

18. Carter, M.; Morris, A.T.; Zhu, F.; Zaluska, W.; Levin, N.W. Effect of body mass index (BMI) on estimation of extracellular volume (ECV) in hemodialysis (HD) patients using segmental and whole body bioimpedance analysis. Physiol. Meas. 2005, 26, S93. [CrossRef]

19. Collins, C.T.; Reid, J.; Makrides, M.; Lingwood, B.E.; McPhee, A.J.; Morris, S.A.; Gibson, R.A.; Ward, L.C. Prediction of body water compartments in preterm infants by bioelectrical impedance spectroscopy. Bioelectrical Impedance Analysis. Eur. J. Clin. Nutr. 2013, 67, S47. [CrossRef]

20. Mazariegos, M.; Klassen, P.; Solomons, N.W.; Fürst, P. Bioelectrical Impedance Spectroscopy in Health and Disease: Correspondence between Whole Body and Segmental Bioelectrical Impedance Spectroscopy Indices in Patients with Classical Dengue Fever. Ann. N. Y. Acad. Sci. 2000, 904, 205. [CrossRef]

21. Gültekin, T.; Dasgupta, P.; Özer, B.K. Segmental Bioelectrical Impedance Analysis in Children Aged 7-18 Years Living in Ankara-Turkey: Age and Sex Difference in the Measures of Adiposity. Pap. Anthropol. 2014, 23, 23-36. [CrossRef]

22. Jiménez, A.; Omaña, W.; Flores, L.; Coves, M.J.; Bellido, D.; Perea, V.; Vidal, J. Prediction of Whole-Body and Segmental Body Composition by Bioelectrical Impedance in Morbidly Obese Subjects. Obes. Surg. 2012, 22, 587. [CrossRef] 
23. Yamada, Y.; Masuo, Y.; Yokoyama, K.; Hashii, Y.; Ando, S.; Okayama, Y.; Morimoto, T.; Kimura, M.; Oda, S. Proximal electrode placement improves the estimation of body composition in obese and lean elderly during segmental bioelectrical impedance analysis. Eur. J. Appl. Physiol. 2009, 107, 135-144. [CrossRef] [PubMed]

24. Jaffrin, M.Y.; Morel, H. Measurements of body composition in limbs and trunk using a eight contact electrodes impedancemeter. Med. Eng. Phys. 2009, 31, 1079-1086. [CrossRef]

(C) 2019 by the authors. Licensee MDPI, Basel, Switzerland. This article is an open access article distributed under the terms and conditions of the Creative Commons Attribution (CC BY) license (http://creativecommons.org/licenses/by/4.0/). 